Trauma Berufskrankh 2018·20 (Suppl 1):S67-S72 https://doi.org/10.1007/s10039-017-0302-6 Online publiziert: 13 . September 2017 (c) Der/die Autor(en) 2017. Dieser Artikel ist eine Open-Access-Publikation.

CrossMark

\author{
S. Hungerer ${ }^{1,2} \cdot$ P. Ziegler ${ }^{3} \cdot$ V. Bühren ${ }^{1} \cdot$ J. Friederichs ${ }^{1}$ \\ 'Abteilung für Gelenkchirurgie und Endoprothetik, BG Unfallklinik Murnau, Murnau, Deutschland \\ ${ }^{2}$ Institut für Biomechanik, Paracelsus Medical University PMU, Salzburg, Österreich \\ ${ }^{3}$ Traumatologie und Rekonstruktive Chirurgie, BG Unfallklinik Tübingen, Tübingen, Deutschland
}

\title{
Nekrotisierende Weichteilinfekte
}

\section{Klinische Diagnose und Therapie- entscheidung}

im Zeitraum von 1980 bis 2000 noch durchgehend bei $20-32 \%$ lag, konnte durch eine Verbesserung der Therapie eine signifikante Reduktion der Letalität auf unter $20 \%$ erreicht werden $[6,9,10$, 20]. Neben der antibiotischen Therapie ist die verbesserte chirurgische Therapie ein Hauptgrund für diesen Fortschritt.

\section{Einteilung}

Eine einfache Einteilung klassifiziert anhand der beteiligten Weichteilstrukturen (Haut und Subkutis, Faszie, Muskel). Als Faustregel gilt hier, je mehr Barrieren durchbrochen sind, desto schlechter die Prognose. Zwei weitere Klassifizierungen von NSSTIs erfolgen anhand histologischer Kriterien einerseits und anhand der Mikrobiologie. Diese Untersuchungen folgen retrospektiv, d. h. sie können zur initialen Therapieentscheidung nicht beitragen. Histologisch zeichnet sich die Erkrankung am häufigsten durch eine fortschreitende Nekrose des Subkutangewebes und der Faszien mit Thrombosierung der mikrovaskulären Gefäße aus. Diese histologischen Merkmale fanden 1979 Eingang in die Definition der nekrotisierenden Fasziitis anhand der sog. Fisher-Kriterien [3]. Diese Kriterien sind einerseits klinische, andererseits diagnostische Faktoren und der erste Versuch, die nekrotisierende Fasziitis zu definieren. Von den 6 Diagnosekriterien müssen 5 zutreffen, um von einer nekrotisierenden Fasziitis zu sprechen [3]. Dargestellt sind die Fisher-Kriterien in - Tab. 1. Ein weiterer Versuch, die nekrotisierenden Weichteilinfekte genauer einzuteilen, stellt die Klassifikation nach Giuliano dar [5]. Giuliano teilte die nekrotisierende Fasziitis in Typ 1 ein, den mit 55-75\% häufigsten Typ, verursacht durch eine Mischinfektion mit grampositiven Kokken, gramnegativen Stäbchen und anaeroben Erregern. Seltener sind Typ-II-Infektionen, die durch einen monomikrobiellen Infekt der Gruppe-A-Streptokokken hervorgerufen werden. Vereinzelt kann auch eine monomikrobielle Staphylococcus-aureus-Infektion vorliegen. Hinzugekommen ist der Typ III durch Infekt mit Vibrio vulnificus, ein Keim der sich in warmem Meerwasser oder rohen Meeresfrüchten findet und in unseren Breiten kaum eine Rolle spielt [15].

Gemeinsam ist diesen Klassifikationen, dass sie erst im Verlauf der Behandlung entstehen und in der klinischen Anfangsphase für die Therapieentscheidungen nutzlos sind. Einen Versuch, bereits in der Initialphase eine Einteilung vorzunehmen, die eine Aussage über die Dringlichkeit der Therapie und die Aggressivität der Erkrankung erlaubt und damit die Therapie nachhaltig beeinflusst, stellt der sog. „Laboratory Risk Indicator for Necrotizing Fasciitis" (LRINEC) dar [21]. Die in - Tab. 2 dargestellten Laborparameter werden mit einer definierten Punktzahl bewertet und in eine Risikokategorie eingeteilt. Dieser Wert lässt dann eine Prognose zu, ob es sich um eine nekrotisierende Weichteilinfektion handelt, und kann zusammen mit anderen diagnostischen Maßnahmen zur Entscheidungsfindung der Therapie beitragen. Der LRINEC ist jedoch wenig bekannt und kommt daher auch kaum zur Anwendung. Zum jet- 


\section{Übersicht}

\section{Tab. 1 Übersicht über die Kriterien der nekrotisierenden Fasziitis nach Fisher [3]}

\begin{tabular}{|c|c|c|}
\hline Nr. & Zeitpunkt & Kriterien \\
\hline 1 & \multirow[t]{2}{*}{ Präoperativ } & Moderates bis schweres septisches Syndrom \\
\hline 2 & & Keine pAVK Grad IV \\
\hline 3 & \multirow[t]{4}{*}{ Postoperativ } & Ausgedehnte Nekrosen der Faszien und des umgebenden Gewebes \\
\hline 4 & & Keine primäre Muskelbeteiligung \\
\hline 5 & & Kein Nachweis von Clostridien in der Mikrobiologie \\
\hline 6 & & $\begin{array}{l}\text { Leukozyteninfiltration } \\
\text { Fokale Fasziennekrose und Nekrose des umgebenden Gewebes } \\
\text { Mikrovaskuläre Thromben }\end{array}$ \\
\hline \multicolumn{3}{|c|}{$\begin{array}{l}\text { Die Kriterien wurden nach dem frühestmöglichen Zeitpunkt der Diagnosestellung sortiert. Es sind } \\
\text { nur } 2 \text { Kriterien sicher vor einem operativen Eingriff zu stellen. Alle anderen Kriterien können erst nach } \\
\text { dem operativen Eingriff erhoben werden. } \\
\text { pAVK periphere arterielle Verschlusskrankheit }\end{array}$} \\
\hline
\end{tabular}

zigen Zeitpunkt fehlt es an einer klinischen Einteilung, die eine therapeutische Konsequenz direkt ableiten lässt und einen chirurgischen und intensivmedizinischen Behandlungsalgorithmus folgen lässt.

\section{Symptome}

Das Kardinalsymptom der nekrotisierenden Weichteilinfekte ist der inadäquate Schmerz (engl.: „pain out of proportion“), der bei über $90 \%$ der Patienten auftritt [1]. Weitere häufige Symptome sind Schwellung, Bläschen, Rötung oder Fluktuationen, die in bis zu $85 \%$ der Fälle vorkommen können. Wesentlich seltener liegen initial septische Symptome wie Fieber, Tachykardie oder Hypotension vor (20-40 \%), Schock und Lymphadenopathien sogar nur bei $15 \%$. Als Konsequenz daraus muss man ableiten, dass jede Haut-Weichteil-Infektion, die mit starken Ruheschmerzen einhergeht, bis zum Beweis des Gegenteils als nekrotisierende Fasziitis gewertet werden muss.

Der initiale Weichteilbefund und die intraoperativen Befunde sollten fotodokumentiert werden (• Abb. 1). Dies ermöglicht, den zeitlichen Verlauf besser zu beurteilen, und hilft, auch Therapieentscheidungen im Nachhinein $\mathrm{zu}$ begründen (• Abb. 2).

\section{Diagnostik}

Das führende Diagnostikum ist weiterhin die klinische Untersuchung. Ergänzt werden muss es durch ein Labor, das die Infektionsparameter Blutbild, C-reaktives Protein (CRP) und PCT (Procalcitonin) beinhalten sollte. Des Weiteren sollte ein Blutbild, der Basenstatus (BE), Blutglukose, Serumnatrium und Kreatinin bestimmt werden. Die erweiterte Diagnostik schließt dann die Leberfunktion, Gerinnung und auch Parameter wie Myoglobin und CK-MM (Creatininkinase Skelett-Muskeltyp) mit ein. Die konventionelle Röntgendiagnostik hat an Bedeutung verloren, mit der inzwischen ubiquitär verfügbaren Computertomographie lassen sich besser Luftansammlungen, Abszesse, Faszienverdickungen oder ein Befall der Muskelschichten nachweisen. Der Einsatz der Magnetresonanztomographie (MRT) wird kontrovers diskutiert, da dem besseren Auflösungsvermögen der Weichteile eine geringe Spezifität entgegensteht. Bei dem akuten Verdacht und einem fulminanten Verlauf ist die langwierige und nicht immer verfügbare MRT-Technik verzichtbar bzw. nicht indiziert.

Ein Ziel der Primäroperation ist die Identifizierung des verursachenden Erregers, um die antibiotische Therapie anpassen zu können. Mehrere aerobe und anaerobe mikrobiologische Proben sollten entnommen werden, um hier die größtmögliche Sicherheit zu bekommen, was manchmal bei polymikrobiellen Infekten sehr schwer ist. Die Gewinnung von histologischen Proben und die histopathologische Aufarbeitung dienen letztendlich nur der Diagnosesicherung und haben in der Akutbehandlung keinen
Tab. 2 Übersicht der Parameter für den

Laboratory Risk Indicator for Necrotizing Fasciitis (LRINEC; [21])

\begin{tabular}{|c|c|c|}
\hline Variable & $\beta$ & Score \\
\hline \multicolumn{3}{|l|}{$C R P(m g / d l)$} \\
\hline$\geq 15$ & 3,5 & 4 \\
\hline \multicolumn{3}{|l|}{ Leukozyten $(/ \mu l)$} \\
\hline $15.000-25.000$ & 0,5 & 1 \\
\hline$>25.000$ & 2,1 & 2 \\
\hline \multicolumn{3}{|l|}{$H b(g / d l)$} \\
\hline $11,0-13,5$ & 0,6 & 1 \\
\hline$<11,0$ & 1,8 & 2 \\
\hline \multicolumn{3}{|l|}{$\mathrm{Na}^{+}(\mathrm{mmol} / \mathrm{l})$} \\
\hline$<135$ & 1,8 & 2 \\
\hline \multicolumn{3}{|l|}{ Kreatinin (mg/dl) } \\
\hline$>1,6$ & 1,8 & 2 \\
\hline \multicolumn{3}{|l|}{ Glukose (mg/dl) } \\
\hline$>180$ & 1,2 & 1 \\
\hline \multicolumn{3}{|c|}{$\begin{array}{l}\text { Ein Wert von } \geq 6 \text { Punkten hat einen } 92 \% \\
\text { positiven prädiktiven Wert. Bei Patienten mit } \\
\text { Diabetes mellitus ist ein Wert von }>8 \text { Punk- } \\
\text { ten sicherer [17]. } \\
\beta \text { Regressionskoeffizient in dem Model [21], } \\
C R P \text { C-reaktives Protein, } H b \text { Hämoglobin }\end{array}$} \\
\hline
\end{tabular}

Stellenwert. Ein Schnellschnittverfahren hat sich nicht durchgesetzt [15]

\section{Differenzialdiagnosen}

Als Differenzialdiagnosen kommen in der Frühphase alle nicht nekrotisierenden Weichteilinfekte in Betracht. Da der Übergang manchmal auch fließend ist, zählen hierzu das Erysipel, die Zellulitis und abszedierende Infektionen wie Furunkel und Abszesse. Auch nichtinfektiöse Prozesse wie Insektenstiche oder Thrombosen/Thrombophlebitiden müssen differenzialdiagnostisch in Betracht gezogen werden.

Sehr schwierig und von therapeutischer Herausforderung ist die Unterscheidung von NSSTIs zum Pyoderma gangraenosum, einer Autoimmunerkrankung, die im klinischen Erscheinungsbild ähnlich verlaufen kann mit septischen Krankheitsbild und begleitenden Weichteilnekrosen mit Eiter (- Abb. 3). Die Therapie ist jedoch diametral zu den NSSTIs - beim Pyoderma gangraenosum ist die Immunsuppression die Hauptsäule. Die frühzeitige therapeutische Weichenstellung ist her- 
ausfordernd, und erst der Therapierfolg sichert die Diagnose des Pyoderma gangraenosum [7].

\section{Klinische Einteilung}

Die oben beschriebenen Einteilungen der NSSTIs sind in der klinischen Praxis nicht hilfreich, da die initial entscheidenden Therapieschritte ohne Wissen der Mikrobiologie und Histologie getroffen werden müssen. Der Verdacht auf eine NSSTI erfordert eine unmittelbare chirurgische Therapie in Kombination mit einer zunächst empirischen 3-fachen Antibiotikatherapie bis zum Erhalt des Keimspektrums und Antibiogramms. Hinzu kommen adjuvante intensivmedizinische Maßnahmen und ggf. auch die hyperbare Sauerstofftherapie.

Die Indikationsstellung bei kreislaufinstabilen Patienten ist klar. Der kardiopulmonal instabile Patient mit Zeichen eines septischen Schocks bedarf eines sofortigen chirurgischen Notfalleingriffes. Schwieriger ist die Indikationsstellung bei einem noch kardiopulmonal stabilen Patienten mit Verdacht auf eine NSSTI. Wir sehen hier dennoch eine dringliche Operationsindikation innerhalb von 6 h. Dies gibt noch die Möglichkeit, den Patienten adäquat vorzubereiten, Risikofaktoren $\mathrm{zu}$ adressieren und mit der antibiotischen Therapie zu starten und somit eine höhere Operationssicherheit zu erlangen. Allerdings muss der Patient klinisch engmaschig überwacht werden (Intensivstation, Intermediate Care), um auf eine rasche Progredienz des Infektgeschehens sofort reagieren zu können. Ein vereinfachter klinischer Algorithmus zum Zeitpunkt der chirurgischen Therapie ist in - Abb. 4 dargestellt.

In unserer Klinik sind mehr als $80 \%$ der Patienten Verlegungen aus der erstbehandelnden Klinik. Die Ankündigung eines Patienten mit nekrotisierender Fasziitis ist vergleichbar mit der Meldung eines Polytraumapatienten. Der Patient wird interdisziplinär mit Schockraumteam empfangen, und die weiteren Therapiemaßnahmen werden unmittelbar festgelegt. Die erforderlichen Ressourcen können gemeinsam abgeschätzt werden, und dies kann der entscheidende Zeitgewinn sein, speziell für die Patienten

Trauma Berufskrankh 2018 20 (Suppl 1):S67-S72 https://doi.org/10.1007/s10039-017-0302-6 (c) Der/die Autor(en) 2017. Dieser Artikel ist eine Open-Access-Publikation.

\section{S. Hungerer $\cdot$ P. Ziegler $\cdot$ V. Bühren $\cdot$ J. Friederichs}

\section{Nekrotisierende Weichteilinfekte. Klinische Diagnose und Therapieentscheidung}

\section{Zusammenfassung}

Unter den nekrotisierenden Haut- und Weichteilinfekten („necrotizing skin and soft tissue infections" [NSSTIs]) wird eine Entität seltener Infektionserkrankungen zusammengefasst, die ein breites Spektrum an klinischen Erscheinungsbildern bieten. Diese reichen von oberflächlichen Hautnekrosen ohne systemische Infektzeichen bis zur klassischen nekrotisierenden Fasziitis mit begleitender fulminanter Sepsis und einer Dynamik, die innerhalb von Stunden zum Tode führen kann. Eine international einheitliche Klassifikation gibt es nicht. Charakteristisch sind die Infektion und das klinische und histologische Bild der Nekrosen der beteiligten Weichteilschichten, ausgelöst durch unterschiedliche Bakterien als monooder polymikrobielle Infektion. Das klinische Erscheinungsbild reicht von subakuten bis fulminanten Verläufen. Entscheidend für die
Prognose bei den fulminant verlaufenden NSSTIs sind daher die richtige Einschätzung und Diagnosestellung. Diese erfolgt initial ohne Kenntnis des Keimspektrums, ohne genaue Kenntnis der betroffenen Weichteilschichten und ohne histologische Sicherung der Diagnose. Daher kommt der ersten klinischen Diagnosestellung eine wichtige Bedeutung zu, da diese auch für die Therapieentscheidungen Weichen stellt. Im Folgenden sollen die klinische Diagnose, Differenzialdiagnose, Therapieoptionen und Prognosefaktoren der NSSTIs praxisnah dargestellt werden.

\section{Schlüsselwörter}

Infektionserkrankung · nekrotisierende Weichteilinfektionen · Nekrotisierende Fasziitis $\cdot$ Pyoderma $\cdot$ Sepsis $\cdot$ Bakterien

\section{Necrotizing soft tissue infections. Clinical diagnosis and therapy decisions}

\section{Abstract}

Necrotizing skin and soft tissue infections (NSSTIs) are a group of rare infections with a diversity of clinical presentations. These range from superficial skin necrosis without systemic inflammatory reactions up to the classical necrotizing fasciitis with a foudroyant septic clinical course. An internationally consistent classification of NSSTIs is nonexistent. These infections are characterized by the clinical and histological picture of necrosis of the involved soft tissues, which is triggered by monomicrobial and polymicrobial infections. The clinical course is characterized by subacute inflammatory reactions up to foudroyant septic progression. Crucial for the prognosis of these foudroyant septic infections is the early and correct assessment, diagnosis and therapy. This initial assessment takes place when there is still no knowledge of the pathogens, the depth of soft tissue layers involved and without histological confirmation of the diagnosis; nevertheless, this intial decision sets the course for the outcome and prognosis of these patients. This article describes the practical aspects of the clinical diagnosis of NSSTIs, the differential diagnosis, prognostic factors and therapeutic options.

\section{Keywords}

Infectious diseases - Necrotizing soft tissue infection - Necrotizing fasciitis - Pyoderma . Sepsis · Bacteria mit einem fulminanten Verlauf der Erkrankung.

\section{Therapie}

Die chirurgische Therapie der NSSTIs muss radikal erfolgen im Sinne „life before limb“. Die Entscheidung, wie ausgedehnt die chirurgischen Maßnahmen ausfallen, hängt vom intraoperativen Befund $\mathrm{ab}$ und kann nur in der Operation getroffen werden (•Abb.5). Diese Entscheidung ist eine Teamentscheidung von Chirurgie und Anästhesie. Die nachfolgend aufgeführten Faktoren werden immer wesentlich in den Entscheidungsprozess einfließen. 


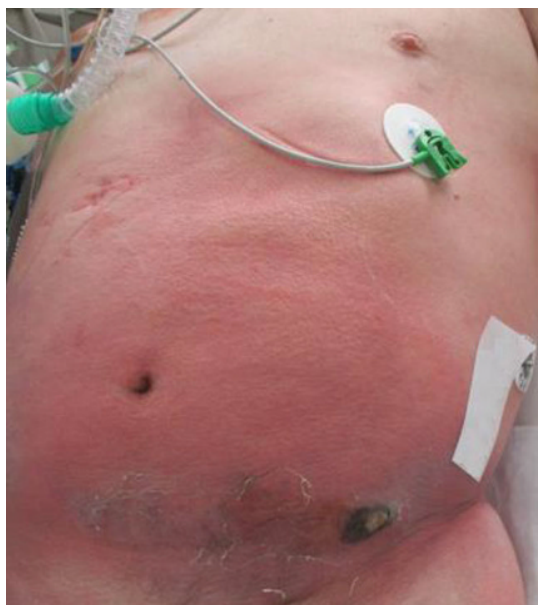

Abb. 1 A Klinisches Bild einer stammbetonten nekrotisierenden Fasziitis bei einem kardiopulmonal instabilen Patienten. Fulminanter Verlauf mit Entwicklung der Klinik innerhalb von $6 \mathrm{~h}$

\section{Ausdehnung der nekrotisierenden Weichteilinfektion}

Die Ausdehnung der nekrotisierenden Weichteilinfektion ist der wesentliche Entscheidungsfaktor, da davon die Prognose des Patienten abhängt. Sind isoliert die Extremitäten betroffen, ist ein radikales Débridement immer möglich, im schlechtesten Falle durch Amputation oder Exartikulation der betroffenen Extremität. Dies kann bei zirkulären ausgedehnten Nekrosen erforderlich werden. Die Letalität steigt sprunghaft, wenn der Infekt den Körperstamm betrifft [4, 13], da dann ein radikales Débridement technisch limitiert sein kann.

\section{Systemic Inflammatory Response Syndrome (SIRS)/Sepsis}

Der Schweregrad der begleitenden systemischen Infektreaktion ist der zweite wesentliche Entscheidungsfaktor. Je instabiler der Patient, desto radikaler muss das Débridement erfolgen, desto eher wird die Indikation zur Amputation der betroffenen Extremität gestellt („life before limb“). Limitierend ist auch hier wieder die Ausdehnung am Körperstamm.

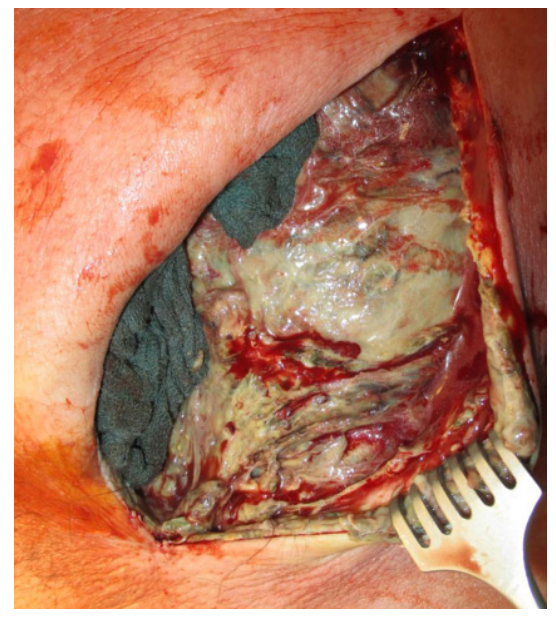

Abb. 2 A Intraoperativer Befund der Faszien im Bereich der oberflächlichen Nekrosen. Weitreichende Nekrosen über die gesamten abdominalen Muskelfaszien auch nach kontralateral und thorakal. Exitus $3 \mathrm{~h}$ nach operativer Sanierung des Infektfokus einer gedeckt perforierten Sigmadivertikulitis und ausgiebigen Débridements der Stammfaszien

\section{Risikofaktoren}

Risikofaktoren sind ein Teil der Entscheidungskriterien für die weitere Therapie. Je älter der Patient und je mehr Begleiterkrankungen und Risikofaktoren er mitbringt, desto kritischer müssen die möglichen Therapieoptionen hinterfragt werden. Einerseits ist eine frühe radikale Operation erforderlich, um eine Sanierung des Infektes erreichen zu können. Andererseits erhöhen einige Faktoren das intraoperative Risiko - insbesondere das Risiko eines großen Eingriffes - und vermindern die Überlebenschance und die Möglichkeit einer chirurgischen Sanierung. Zu den Risikofaktoren zählen Alter, Immunsuppression, Niereninsuffizienz, Katecholaminbedarf, Injektionen als Eintrittspforte, Adipositas, klinische Dynamik der Infektion $[4,20]$.

Die chirurgische Therapie richtet sich weitestgehend nach dem klinischen und intraoperativen Befund. Die Befunde müssen fotodokumentiert werden (- Abb. 2). Alle nekrotischen Weichteile sollten entfernt werden. Dies kann bei den NSSTIs nur die Haut und Subkutis betreffen, bei Befall der Faszien müssen die betroffenen und eitrig belegten Faszien komplett debridiert werden (• Abb. 5). Zeigen sich auch Muskelschichten avital und nicht durchblutet, müssen auch diese reseziert werden. Sind alle Weichteile einer Extremität tief reichend und zirkulär betroffen, kommt zur Sanierung des Infektes nur noch die Amputation der betroffenen Extremität infrage. Es sollten aus den betroffenen Schichten wenigsten 4 mikrobiologische und histologische Proben gewonnen werden.

Nach Resektion der Weichteile bietet sich die negative Unterdrucktherapie („negative wound pressure therapy“ [NWPT]) zur temporären Weichteildeckung an. Die NWPT erlaubt einen sterilen Verband großflächiger Wunden mit gutem Management des Sekretes. Die NWPT eignet sich auch für den gesamten Körperstamm und reduziert die Wahrscheinlichkeit bakterieller Superinfektionen.

Der „second look“ - d.h. die Revisionsoperation - sollte innerhalb von 24-48 h erfolgen, bestenfalls durch denselben Chirurgen. Sie ist erforderlich zur Prognoseabschätzung, Nachresektion von weiteren Nekrosen und erneuter Probengewinnung für die Mikrobiologie/Histologie.

\section{Prognose}

Wie oben beschrieben, ist eine sofortige radikale chirurgische Therapie der Schlüssel zum Erfolg. In Studien konnte gezeigt werden, dass das frühzeitige chirurgische Vorgehen eine starker prognostischer Faktor ist und eine Verzögerung der chirurgischen Therapie eine höhere Mortalität und eine höhere Amputationsrate verursacht $[2,8,10$, 12, 16]. Dies konnte auch für die Zeit zwischen Krankenhauseinweisung und erster Operation gezeigt werden [10]. Da diese Faktoren durch die behandelnden Ärzte beeinflusst werden können, halten wir die notfallmäßige Triage eines jeden Patienten mit dem Verdacht auf eine NSSTI für zwingend, um im Sinne eines Polytraumas schnellstmöglich handeln zu können. Patienteneigene prognostische Faktoren wie hohes Alter, weibliches Geschlecht, Begleiterkrankungen wie Diabetes mellitus können vom Behandler nicht beeinflusst werden. Es ist bekannt, dass eine nekrotisierende Weichteilinfektion des Körperrumpfes 

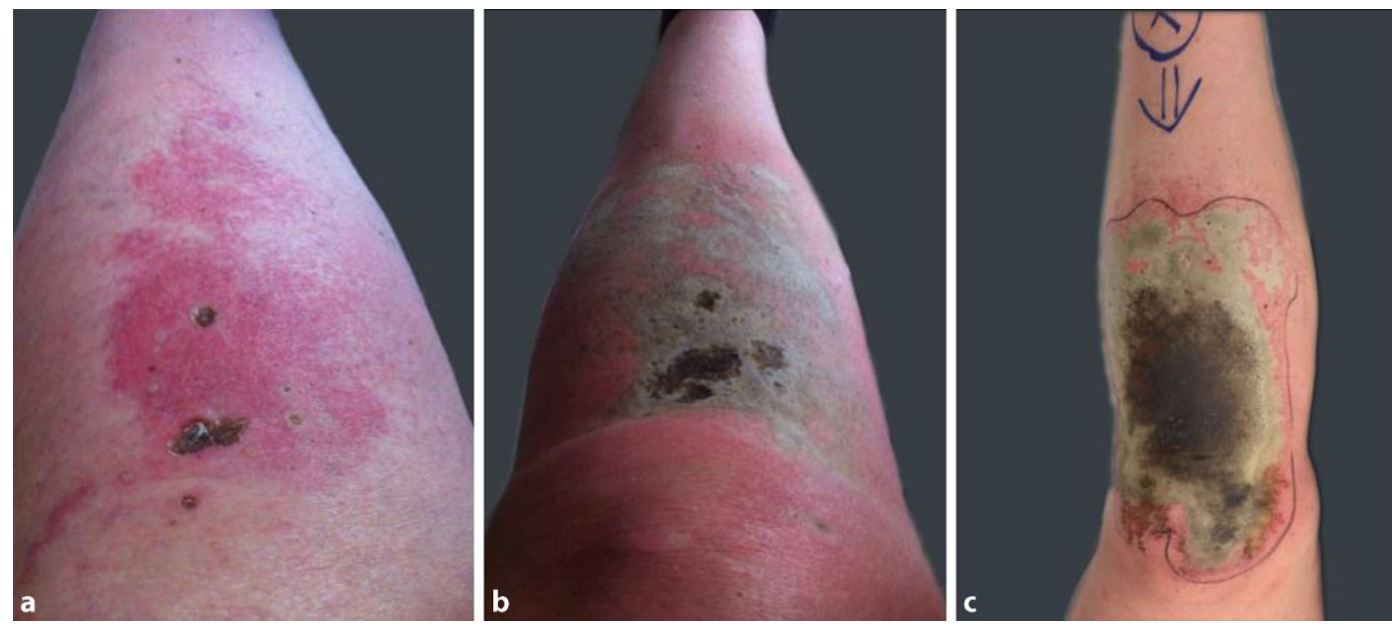

Abb. $3<$ Differenzialdiagnose Pyoderma gangraenosum. Fraglicher Auslöser Insektenstich an der Tibiavorderkante a Tag 2, $\mathbf{b}$ Tag 4 und c Tag 6. Begleitend geringe systemische Infektzeichen am Tag 6. Histologisch vereinbar mit inzipientem Pyoderma gangraenosum. Mikrobiologie war in 6 Proben negativ

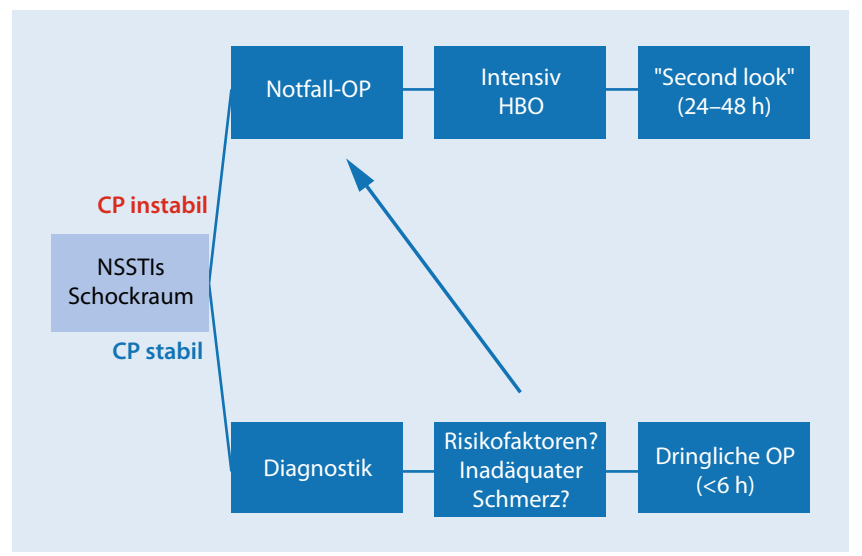

Abb. 4 ム Klinischer Algorithmus der "necrotizing skin and soft tissue infections" (NSSTIs): Unterscheidung anhand der kardiopulmonalen Situation des Patienten. Der kardiopulmonal instabile Patient ist eine absolute Notfallindikation zum chirurgisch radikalen Débridement. Der kardiopulmonal stabile Patient erlaubt noch diagnostische Maßnahmen und Optimierung der Operationsfähigkeit, sollte aber als dringliche Operation (OP) geplant werden. $C P$ kardiopulmonal, $H B O$ hyperbare Sauerstofftherapie

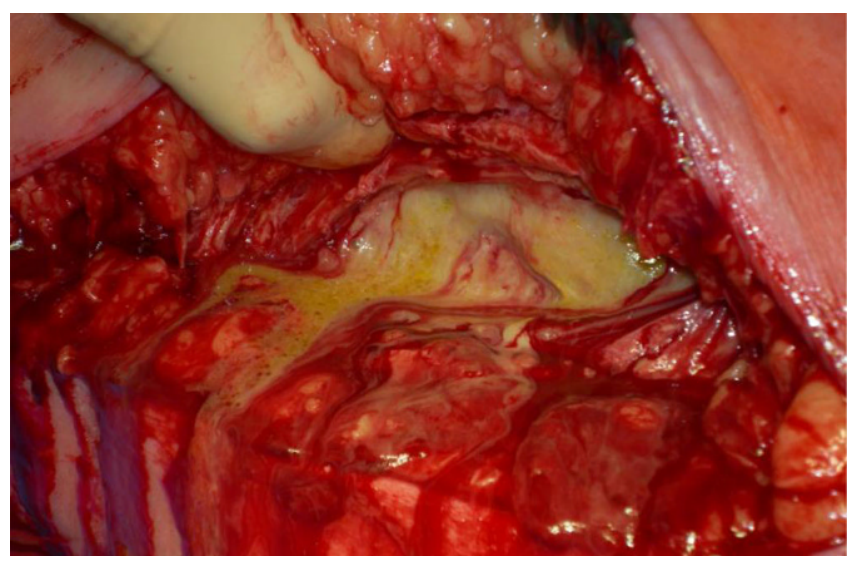

Abb. $5<$ Intraoperativer Befund einer nekrotisierenden Fasziitis mit putridem Sekret auf den Faszien mit einer schlechteren Prognose verbunden ist [13], ebenso konnte für die nekrotisierende Fasziitis nach Injektionstherapie eine deutlich höhere Amputationsrate und Mortalität festgestellt werden [4]. Die Kenntnis dieser unterschiedlichen prognostischen Gegebenheiten zum Zeitpunkt der stationären Aufnahme in Verbindung mit dem akutklinischen Bild und dem kurzfristigen Verlauf sollte Basis für eine schnelle und chirurgische Entscheidung sein.

Nach der initialen chirurgischen Therapie stehen die intensivmedizinische Komplextherapie und die antibiotische Therapie im Vordergrund. Die Notwendigkeit einer adjuvanten hyperbaren Sauerstofftherapie wird in der Literatur kontrovers diskutiert [14, 19], der Nachweis der Wirksamkeit oder Unwirksamkeit des Verfahrens steht noch aus. Gerade bei Unkenntnis des Erregerspektrums und des $\mathrm{zu}$ erwartenden Nutzens bei anaeroben Keimen ist unserer Meinung nach bei fehlenden Kontraindikationen eine adjuvante hyperbare Sauerstofftherapie sinnvoll und anzuraten. Es muss jedoch sichergestellt sein, dass hierdurch keine Verzögerung der chirurgischen Therapie z. B. durch aufwendige Verlegungen und damit eine Prognoseverschlechterung erreicht wird.

\section{Fazit für die Praxis}

\section{- Die initiale Diagnose der nekrotisie- renden Weichteilinfektion ist eine rein klinische Diagnose. Begleiten- de Risikofaktoren können weitere Indizien für die Diagnose sein.}


- Leitsymptom der nekrotisierenden Weichteilinfektionen ist der unverhältnismäßige Schmerz. Die Übergänge von einfachen Weichteilinfekten zu den nekrotisierenden Weichteilinfektionen können flieBend sein. Eine sofortige operative Exploration ist bei Verdacht indiziert.

- Nekrotisierende Weichteilinfekte können initial schwierig einzuschätzen sein und sind das „Polytrauma” der Weichteilinfektionen. Eine interdisziplinäre Einschätzung und Therapie in Analogie zum Polytraumamanagement des Erstbefundes sind erforderlich, da der Faktor Zeit für die Prognose entscheidend ist.

- Eine Fotodokumentation der Befunde ist zwingend erforderlich.

\section{Korrespondenzadresse}

\section{PD Dr. S. Hungerer}

Abteilung für Gelenkchirurgie und Endoprothetik, BG Unfallklinik Murnau Professor-Küntscher-Str. 8, 82418 Murnau, Deutschland

sven.hungerer@bgu-murnau.de

Acknowledgements. Open access funding provided by Paracelsus Medical University.

\section{Einhaltung ethischer Richtlinien}

Interessenkonflikt. S. Hungerer, P. Ziegler, V. Bühren und J. Friederichs geben an, dass kein Interessenkonflikt besteht.

Dieser Beitrag beinhaltet keine von den Autoren durchgeführten Studien an Menschen oder Tieren.

The supplement containing this article is not sponsored by industry.

Open Access. Dieser Artikel wird unter der Creative Commons Namensnennung 4.0 International Lizenz (http://creativecommons.org/licenses/by/4.0/deed. de) veröffentlicht, welche die Nutzung, Vervielfältigung, Bearbeitung, Verbreitung und Wiedergabe in jeglichem Medium und Format erlaubt, sofern Sie den/die ursprünglichen Autor(en) und die Quelle ordnungsgemäßnennen, einen Linkzur Creative Commons Lizenz beifügen und angeben, ob Änderungen vorgenommen wurden.

\section{Literatur}

1. Childers BJ, Potyondy LD, Nachreiner R et al (2002) Necrotizing fasciitis: a fourteen-year retrospective study of 163 consecutive patients. Am Surg 68:109-116
2. Elliott DC, Kufera JA, Myers RA (1996) Necrotizing soft tissue infections. Risk factors for mortality and strategies formanagement. AnnSurg 224:672-683

3. Fisher JR, Conway MJ, Takeshita RT et al (1979) Necrotizing fasciitis. Importance of roentgenographic studies for soft-tissue gas. JAMA 241:803-806

4. Friederichs J, Torka S, Militz M et al (2015) Necrotizing soft tissue infections after injection therapy: Higher mortality and worse outcome compared to other entry mechanisms. J Infect 71:312-316

5. Giuliano A, Lewis F Jr., Hadley K et al (1977) Bacteriology of necrotizing fasciitis. Am J Surg 134:52-57

6. Gunter OL, Guillamondegui OD, May AK et al (2008) Outcome of necrotizing skin and soft tissue infections. Surg Infect (Larchmt) 9:443-450

7. HackI S, Merkel P, Hungerer Set al (2015) Pyoderma gangrenosum after intramedullary nailing of tibial shaft fracture: A differential diagnosis to necrotizing fasciitis. Unfallchirurg 118:1062-1066

8. Herr M, Grabein B, Palm HG et al (2011) Necrotizing fasciitis. 2011 update. Unfallchirurg 114:197-216

9. Hsiao CT, Weng HH, Yuan YD et al (2008) Predictors of mortality in patients with necrotizing fasciitis. Am JEmerg Med 26:170-175

10. Kao LS, Lew DF, ArabSN etal (2011) Local variations in the epidemiology, microbiology, and outcome of necrotizing soft-tissue infections: a multicenter study. Am J Surg 202:139-145

11. Lamagni TL, Darenberg J, Luca-Harari B et al (2008) Epidemiology of severe Streptococcus pyogenes disease in Europe. J Clin Microbiol 46:2359-2367

12. Malangoni MA (2001) Necrotizing soft tissue infections: are we making any progress? Surg Infect (Larchmt) 2:145-150 (discussion 150-142)

13. Paty R, Smith AD (1992) Gangrene and Fournier's gangrene. Urol Clin North Am 19:149-162

14. Schmale M, Fichtner A, Pohl C et al (2012) Hyperbaric oxygenation for necrotizing soft tissue infections: pro. Chirurg 83:973-979

15. Schnurer S, Beier JP, Croner Ret al (2012) Pathogenesis, classification and diagnosis of necrotizing soft tissue infections. Chirurg 83:943-952

16. Schwartz S, Kightlinger E, De Virgilio C et al (2013) Predictors of mortality and limb loss in necrotizing soft tissue infections. Am Surg 79:1102-1105

17. Tan JH, Koh BT, Hong CC et al (2016) A comparison of necrotising fasciitis in diabetics and nondiabetics: a review of 127 patients. Bone Joint J 98B:1563-1568

18. Ustin JS, Malangoni MA (2011) Necrotizing softtissue infections. Crit Care Med 39:2156-2162

19. Willy C, Rieger H, Vogt D (2012) Hyperbaric oxygen therapy for necrotizing soft tissue infections: contra. Chirurg 83:960-972

20. Wong $\mathrm{CH}$, Chang HC, Pasupathy $\mathrm{S}$ et al (2003) Necrotizing fasciitis: clinical presentation, microbiology, and determinants of mortality. J Bone Joint Surg Am 85-A:1454-1460

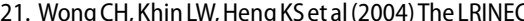
(Laboratory Risk Indicator for Necrotizing Fasciitis) score: a tool for distinguishing necrotizing fasciitis from other soft tissue infections. Crit Care Med 32:1535-1541 